\title{
« Das Bier unter den Alkoholfreien »- Mensonge et tromperie dans la communication commerciale
}

"Das Bier unter den Alkoholfreien" - Lüge und Irreführung in der kommerziellen Kommunikation

"Das Bier unter den Alkoholfreien" - lying and misleading practices in commercial communication

\section{Nathalie Schnitzer}

\section{OpenEdition}

\section{Journals}

Édition électronique

URL : http://journals.openedition.org/ceg/1482

DOI : $10.4000 /$ ceg. 1482

ISSN : 2605-8359

\section{Éditeur}

Presses Universitaires de Provence

Édition imprimée

Date de publication : 15 juin 2015

Pagination : 117-130

ISBN : 978-2-85399-993-9

ISSN : 0751-4239

\section{Référence électronique}

Nathalie Schnitzer, « «Das Bier unter den Alkoholfreien » - Mensonge et tromperie dans la

communication commerciale ", Cahiers d'Études Germaniques [En ligne], 68 | 2015, mis en ligne le 17 décembre 2017, consulté le 02 décembre 2020. URL : http://journals.openedition.org/ceg/1482 ; DOI : https://doi.org/10.4000/ceg.1482 


\section{« Das Bier unter den Alkoholfreien » - Mensonge et tromperie dans la communication commerciale}

Nathalie SCHNITZER

Aix Marseille Université

Cette contribution relève le défi d'aborder dans une perspective linguistique un phénomène qui n'est pas inscrit en langue, mais seulement en discours. L'analyse s'appuie sur un corpus français et allemand et sur une conception pragmatique du mensonge, défini comme un acte de langage consistant à affirmer ce que l'on croit faux dans le but de tromper l'interlocuteur. La réflexion est étayée par des exemples relevant de la communication commerciale, qu'il s'agisse de slogans publicitaires, de noms de produits ou de marques, ou encore d'appellations d'origine protégée (AOP) et d'indications géographiques protégées (IGP) régies par la réglementation européenne.

\section{Mensonge et langue}

Défensif ou offensif, altruiste ou égoïste, moralement condamnable ou humainement excusable, le mensonge possède de multiples facettes. Pour le linguiste, étudier le mensonge est une entreprise risquée dans la mesure où il n'existe pas de marqueurs linguistiques spécifiques du mensonge comme on pourrait parler de marqueurs de l'hypothèse, de l'interrogation, de la concession ou de la subjectivité du locuteur. Comme le souligne l'anthropologue Gérard Lenclud, le mensonge s'appuie sur les mêmes conventions linguistiques que l'assertion sincère: « C'est pourquoi, même s'il revêt un habit langagier, le mensonge ne relève pas de l'analyse du linguiste, en tout cas pas de celui traitant du langage comme d'un code » (LENCLUD 2011 : 4). Le linguiste Franz Hundsnurscher ne dément pas, mais précise :

[...] solange die Sprachwissenschaft ihr Erkenntnisinteresse ausschließlich auf zeichenhaft-funktionale Aspekte der Äußerungsformen, d.h. auf die Beschreibung der Struktur und Systemeigenschaften der sprachlichen Mittel nach grammatischen Kategorien beschränkte, konnte die Lüge nicht sinnvoll untersucht und auch nichts Nennenswertes über sie herausgefunden werden, eben weil Lügen kein grammatisches Phänomen ist (HuNDSNURSCHER 1994: 98).

Le mensonge ne devient objet d'étude pour le linguiste que dans le cadre d'une description pragmatique du discours prenant en compte non seulement le message 
en tant que tel (l'énoncé comme produit fini), mais aussi les circonstances de son énonciation, les interlocuteurs et le contexte. C'est à ce niveau, pragmatique, que se situent les indices de la présence du mensonge. La conception du mensonge adoptée ici est formulée de manière synthétique par Anne Reboul:

(i) mentir, c'est dire ce que l'on croit faux;

(ii) le menteur a l'intention de tromper son interlocuteur (ReBoul 1992: 131)

Elle fait écho à celle proposée par Bettina Kümmerling-Meibauer et Jörg Meibauer:

(i) Lügen ist ein Sprechakt, bei dem der Sprecher etwas behauptet, an dessen Wahrheit er nicht glaubt. (ii) Lügen ist verbunden mit der Intention, den Hörer zu täuschen. (Der Sprecher ist nicht wahrhaftig.) (iii) Ein Sprecher lügt, wenn er die Absicht hat zu täuschen, auch wenn er zufällig die Wahrheit gesagt hat. (KüMmERLING-MeIBAUER, MeIBAUER 2011: 115)

Il est remarquable que la notion de vérité ne soit pas directement invoquée pour définir cet acte de langage. En effet, le mensonge ne s'oppose pas à une vérité objective, factuelle, dont il prendrait l'exact contrepied. D'une part la vérité n'est pas toujours clairement établie, d'autre part il arrive au menteur de dire la vérité sans le savoir. Le mensonge est en revanche étroitement lié à la conscience du locuteur, à ses propres croyances et à une intention de communication, cette intention trompeuse que la morale condamne, à quelques exceptions près. On tolère généralement que le locuteur énonce ce qu'il croit faux pour se protéger, sauver sa vie ou celle d'autrui, ou plus couramment pour se ménager ou ménager autrui. Inversement, l'énonciation sincère n'est pas toujours motivée par une intention louable. Dire à quelqu'un ce que l'on croit vrai, alors que, dans certaines circonstances, il vaudrait mieux se taire, peut relever de la volonté de nuire, voire de la délation.

\begin{tabular}{|c|c|c|c|}
\cline { 2 - 4 } \multicolumn{1}{c|}{} & $\begin{array}{c}\text { intention } \\
\text { de tromper }\end{array}$ & intention de nuire & $\begin{array}{c}\text { adéquation } \\
\text { avec la vérité }\end{array}$ \\
\hline énoncé sincère & - & $?$ & \\
\hline énoncé mensonger & + & $?$ & $?$ \\
\hline
\end{tabular}

La définition du mensonge qui vient d'être proposée ne s'appuie pas sur des critères moraux et le linguiste doit se garder de toute attitude moralisatrice, même lorsqu'il a affaire à un corpus publicitaire dont l'affinité avec le mensonge ne fait pas de doute. Le rôle de l'analyste n'est pas de disqualifier ce type de discours en général, comme le souligne très justement Nina Janich, mais d'en déceler les ressorts cachés :

Statt vor Manipulation zu warnen, vergeblich Information einzufordern und nicht informative Werbung moralisch zu verurteilen, könnte sprachwissenschaftlich begründete Aufklärung darin liegen, möglichst wertungsfrei die werbesprachlichen Überzeugungsstrategien zu beschreiben, den intentionalen Charakter von Werbung zu betonen, die Inszeniertheit ihrer Sprache bewusst zu machen und auf die Selektivität und Subjektivität der Produktinformationen hinzuweisen. (JANICH 1999: 35) 
Les réflexions qui suivent s'inscrivent dans cette perspective et visent une meilleure compréhension du fonctionnement du mensonge dans le domaine $d u$ marketing et de la publicité, des activités dont on sait qu'elles ont pour fonction première d'augmenter le chiffre d'affaire des entreprises et non de fournir des informations objectives et désintéressées au consommateur et client potentiel.

En ce qui concerne le vecteur linguistique du mensonge, on a coutume de l'associer à la forme assertive, c'est-à-dire à une proposition qui peut être dite vraie ou fausse. Kümmerling-Meibauer et Meibauer utilisent d'ailleurs le verbe « behaupten » pour décrire l'action du menteur. Or si le mensonge peut à la limite se passer de tout vecteur linguistique (mensonge par omission), sa capacité à se fixer sur un simple mot, un groupe de mots, une phrase nominale, ou encore dans une phrase verbale non assertive est rarement évoquée; peut-être parce que la force du mensonge est d'être toujours là où on ne l'attend pas. C'est ce que l'analyse du corpus tentera de mettre en évidence.

\title{
Éléments de législation
}

Dans un souci d'harmonisation, l'Union européenne s'est progressivement dotée d'outils législatifs permettant de lutter contre ce qu'on appelle communément la « publicité mensongère ». Les deux principales directives actuellement en vigueur, l'une portant sur les pratiques commerciales déloyales, l'autre sur la publicité trompeuse et sur la publicité comparative datent respectivement de 2005 et 2006. La directive du 11 mai 2005 « relative aux pratiques commerciales déloyales des entreprises vis-à-vis des consommateurs » définit précisément ce qu'est une action trompeuse :

\begin{abstract}
Une pratique commerciale est réputée trompeuse si elle contient des informations fausses, et qu'elle est donc mensongère ou que, d'une manière quelconque, y compris par sa présentation générale, elle induit ou est susceptible d'induire en erreur le consommateur moyen, même si les informations présentées sont factuellement correctes, [...] et que, dans un cas comme dans l'autre, elle l'amène ou est susceptible de l'amener à prendre une décision commerciale qu'il n'aurait pas prise autrement (extrait de l'article 6 de la directive 2005/29/(CE ${ }^{1}$ ).
\end{abstract}

Cette directive est complétée par celle du 12 décembre 2006 « en matière de publicité trompeuse et de publicité comparative» et visant la protection des professionnels. On entend par

" publicité trompeuse ", toute publicité qui, d'une manière quelconque, y compris sa présentation, induit en erreur ou est susceptible d'induire en erreur les personnes auxquelles elle s'adresse ou qu'elle touche et qui, en raison de son caractère trompeur, est susceptible d'affecter leur comportement économique ou qui, pour ces raisons, porte préjudice ou est susceptible de porter préjudice à un concurrent (extrait de l'article 2 de la directive 2006/114/CE ${ }^{2}$ ).

1 http://eur-lex.europa.eu/LexUriServ/LexUriServ.do?uri=OJ:L:2005:149:0022:0039:FR:PDF (dernière consultation pour ce lien et les suivants: 10 mai 2014)

2 http://eur-lex.europa.eu/LexUriServ/LexUriServ.do?uri=OJ:L:2006:376:0021:0027:FR:PDF 
On remarque que l'adjectif « trompeur », mis en avant dans les deux directives, n'est pas synonyme de « mensonger ». Un contenu mensonger suppose une intention de tromper, d'induire le consommateur en erreur. Alors qu'un contenu peut produire un effet trompeur sans intention de tromper, simplement par maladresse ou ignorance, et cela même lorsque " les informations présentées sont factuellement correctes ». Pour sa défense, un diffuseur accusé de publicité trompeuse ne pourra donc pas se contenter d'arguer de sa bonne foi ni de la vérité de ses allégations. Le législateur épargne ainsi aux tribunaux la tâche délicate de statuer sur le caractère intentionnel de l'action trompeuse. Il est déjà assez délicat pour le linguiste de décrypter l'intention du locuteur. Mais lorsque le message consiste en une combinaison d'éléments verbaux, visuels, acoustiques, comme c'est souvent le cas en publicité, la tâche n'en est que plus périlleuse et le résultat incertain. Le législateur a donc choisi de ne tenir compte que de l'effet que le message est susceptible de produire sur le récepteur, que cet effet ait été ou non anticipé.

\begin{tabular}{|c|c|c|}
\hline & intention de tromper & effet trompeur \\
\hline contenu trompeur & $?$ & + \\
\hline contenu mensonger & + & + \\
\hline
\end{tabular}

La même précaution, qui consiste à éviter toute référence explicite à la mauvaise foi de l'annonceur, a également été appliquée aux lois transposant ces directives européennes en droit français et en droit allemand. Du côté français, on pourra consulter sur ce point la loi du 4 août 2008 (article L121-1 du Code de la consommation ${ }^{3}$ ). Du côté allemand, on se référera à la loi intitulée "Gesetz gegen den unlauteren Wettbewerb » $\left(\mathrm{UWG}^{4}\right)$, où il est question de « irreführende geschäftliche Handlungen » (§5).

\section{Culture du mensonge en publicité}

Si l'Union européenne s'est dotée ces dernières années d'un véritable arsenal législatif pour lutter contre les pratiques commerciales trompeuses, on aurait tort de voir dans les pratiques qu'elle tente de juguler un effet récent du développement de la société de consommation et de ses excès. Le recours au mensonge en publicité n'est pas une nouveauté, un dévoiement de la publicité des origines, « la réclame », qui aurait été véritablement dédiée à l'information du client. La presse du début $\mathrm{du} \mathrm{XX}^{\mathrm{e}}$ siècle contient déjà nombre de publicités suggestives manifestement destinées à tromper le consommateur. L'étonnante campagne pour la promotion $\mathrm{du}$ médicament Urodonal (LeFÈBVRE 2004 : 680) en fournit un bel exemple. Sur l'une

\footnotetext{
http://www.legifrance.gouv.fr/affichCodeArticle.do;jsessionid=91CB08968D3CB0A6CE9876A 8AE2B8B9B.tpdjo17v_1?idArticle $=$ LEGIARTI000019293636\&cidTexte $=$ LEGITEXT000006069565\& dateTexte $=20131124$

4 http://www.gesetze-im-internet.de/bundesrecht/uwg_2004/gesamt.pdf
} 
des nombreuses annonces publicitaires consacrées à cette potion miracle, on voit un homme confortablement installé dans son fauteuil dans un intérieur bourgeois, il fume un cigare dont s'échappent de belles volutes blanches qui se transforment en silhouettes de femmes aux formes avantageuses et aux décolletés plongeants ${ }^{5}$. Le pavé rédactionnel associé à cette illustration retient également l'attention:

(1) L'Urodonal permet le cigare en supprimant le danger de la nicotine

Songez, fumeurs, au précieux Urodonal. Rappelez-vous qu'il n'est rien de tel pour assouplir les vaisseaux, conserver la tonicité du cœur, abaisser la tension vasculaire, enrayer la sclérose, décrasser le sang, éliminer les toxines, enfin et surtout dissoudre l'acide urique, comme l'eau chaude dissout le sucre; bref, neutraliser au fur et à mesure la néfaste besogne de la nicotine. Il est évident que si deux forces égales pèsent, chacune de son côté, contre une cloison, l'équilibre aura toutes les chances d'être assuré. Voilà comment, avec l'accompagnement d'un verre d'Urodonal, un bon cigare, une bonne pipe, voire même une série de cigarettes, ne sauraient plus désormais faire du mal à personne.

Il y a déjà dans cette publicité suggestive datant de 1918 des outils très performants de manipulation: l'utilisation hors de propos et l'érotisation du corps féminin (dans la partie iconographique), les allégations de santé non vérifiées scientifiquement (dans la partie textuelle). Même si l'on considère qu'on n'avait pas à l'époque la même connaissance qu'aujourd'hui des méfaits du tabac, il semble difficile de nier la présence dans ce message d'une intention de tromper, subordonnée à la volonté de vendre.

Le mensonge ne s'expose plus aujourd'hui de façon aussi outrancière. La législation actuelle sur les médicaments ${ }^{6}$ ne permettrait d'ailleurs plus de proférer de telles allégations de santé. La marge de manœuvre s'étant considérablement réduite, les professionnels de la communication ont recours à des moyens plus indirects pour créer des besoins, faire connaître et valoriser leurs produits, se démarquer de la concurrence et bien sûr pousser les consommateurs vers l'acte d'achat. Les méthodes utilisées s'inscrivent le plus souvent dans une logique d'idéalisation du produit et vont de l'omission à l'hyperbole en passant par l'exagération.

\section{Diversion par omission}

En publicité, il est courant de faire diversion, c'est-à-dire de détourner l'attention des consommateurs des inconvénients, des risques et autres conséquences indésirables liés à l'utilisation d'un produit ou d'un service en mettant l'accent sur ses avantages réels ou supposés. Cette stratégie de l'évitement est même nécessaire pour des produits controversés comme le tabac et les alcools (conséquences pour la santé, dépendance), l'automobile et l'aviation (pollution, bruit), les médicaments (effets secondaires), l'énergie nucléaire (gestion des déchets), les crédits à la

5 http://commons.wikimedia.org/wiki/File:Urodonal_add2.jpg

6 Voir la directive 2001/83/CE du 6 novembre 2001 instituant un code communautaire relatif aux médicaments à usage humain:

http://eur-lex.europa.eu/LexUriServ/LexUriServ.do?uri=CONSLEG:2001L0083:20110120:FR:PDF 
consommation (surendettement), etc. La dissimulation des questions qui fâchent sous un verni flatteur est un classique des slogans publicitaires.

(2) Lufthansa : Verkürzte Reisezeit - verlängerte Ferien! (1961) ${ }^{7}$

(3) Air France: L'art du voyage (1988) ${ }^{8}$

(4) Cabinet: Von Mensch zu Mensch (1995) ${ }^{9}$

(5) Gauloises blondes: Liberté toujours $(2000)^{10}$

La volonté de valoriser le produit sans insister sur ses faiblesses se mue parfois en opération de désinformation. Il y a alors non seulement omission d'une information substantielle, mais le contenu qui vient se substituer au contenu gênant est en outre lui-même trompeur. Dans les années 80 , une campagne pour la cigarette « R1 Slim Line », destinée principalement aux femmes, s'appuie sur la formule d'accroche:

(6) Schlank steht mir! (1987) ${ }^{11}$

Cette exclamative est associée à un visuel montrant une jeune femme mince et élégante fumant cette cigarette. Le slogan « Schlank und geschmackvoll » complète le message. Le terme « schlank », employé dans le slogan et dans l'accroche, réfère aussi bien à l'objet qu'à la personne qui le consomme, ce qui non seulement détourne les destinataires de l'idée de nocivité du tabac, mais les incite en outre à en déduire que la cigarette favorise la minceur, un argument auquel les femmes, le cœur de cible de cette campagne, sont particulièrement sensibles.

La publicité pour les produits du tabac est aujourd'hui interdite au niveau européen ${ }^{12}$, et dans les autres domaines, la législation a été durcie en matière de mensonge par omission que les textes qualifient $d$ ' « omissions trompeuses ${ }^{13}$ ». Cela n'empêche évidemment pas certains annonceurs de contourner les interdits et d'exploiter les failles du système. Dans le secteur alimentaire, c'est notamment le cas pour les sodas dont les publicitaires vantent les qualités rafraichissantes et dynamisantes, en oubliant de préciser qu'elles contiennent de grandes quantités de sucre. Les associations de consommateurs dénoncent régulièrement ces procédés. En France, ce rôle est notamment assumé par l'association «UFC-Que Choisir ${ }^{14}$ ». En Allemagne, l'association «Foodwatch » s'est quant à elle fixé pour seul objectif la lutte contre la publicité mensongère et trompeuse dans le domaine de l'alimentation. Ses membres ont lancé depuis 2009 une campagne médiatique

7 http://www.slogans.de/slogans.php?BSelect[]=283

8 http://memoireairfrance.canalblog.com/archives/2007/05/07/4865108.html

9 http://www.reemtsma.com/images/home/Reemtsma_100-Jahre-Broschuere.pdf

${ }^{10} \mathrm{http} / /$ gauloises-100ans-de-design-de-marque.blogspot.fr/

11 http://www.reemtsma.com/images/phocagallery/Historie/Werbemotive/thumbs/phoca_thumb_1_ R1\%20Slim\%20Line-Schlank\%20steht\%20mir\%20Werbeanzeige\%201987.jpg

12 Voir la directive 2003/33/CE du 26 mai 2003 «concernant le rapprochement des dispositions législatives, réglementaires et administratives des États membres en matière de publicité et de parrainage en faveur des produits du tabac ». http://eur-lex.europa.eu/legal-content/FR/TXT/PDF/?uri=CELEX:320 03L0033\&from $=$ FR

${ }^{13}$ L'omission trompeuse est définie dans la directive européenne 2005/29/CE sur les pratiques commerciales déloyales.

${ }^{14}$ L'UFC-Que Choisir a récemment fait grand bruit en déposant plainte contre l'opérateur Orange à propos de la campagne de publicité pour la technologie $4 \mathrm{G}$. 
intitulée « abgespeist » relayée par un site web sur lequel les internautes peuvent élire la publicité la plus mensongère de l'année (« die dreisteste Werbelüge des Jahres »). L'association remet chaque année le «goldener Windbeutel» à la campagne ayant obtenu le plus grand nombre de suffrages et fait en sorte d'attirer l'attention des médias sur cet événement ${ }^{15}$.

\section{Diversion par délégation}

$\mathrm{Si}$, comme on l'a vu plus haut, le mensonge est un acte de langage lorsqu'il fait l'objet d'une énonciation, il se manifeste également sous des formes non linguistiques. Dans la communication commerciale, la tentation est forte de faire transiter le contenu mensonger par le biais de l'iconographie; le phénomène ne concerne pas seulement les annonces publicitaires, mais aussi les emballages. À côté des contraintes techniques liées au conditionnement, l'emballage constitue en effet un espace de communication gratuit que les marques ont tout intérêt à exploiter. Certaines respectent les limites du cadre légal ${ }^{16}$, d'autres non. En matière de fraude, on pense au récent scandale des lasagnes étiquetées «pur bœuf» qui contenaient en réalité de la viande de cheval ${ }^{17}$. Un procédé plus subtil consiste à agrémenter l'emballage d'images flatteuses d'ingrédients que le produit ne contient qu'en quantité infime ou sous forme d'arômes. Le packaging trompeur de la tisane «Landlust Mirabelle \& Birne» de la marque Teekanne a ainsi été dénoncé par l'association Foodwatch:

«Genießen Sie einen kleinen Ausflug aufs Land und entdecken Sie den ursprünglichen Genuss vertrauter Früchte, die noch in Ruhe heranreifen können ", verspricht TEEKANNE auf der Verpackung des Früchtetees «Landlust Mirabelle \& Birne », auf der goldgelbe Mirabellen und Birnen prangen. Doch genau genommen ist das Produkt ein ganz normaler Industrie-Früchtetee: Billige Standard-Zutaten plus Aroma für imitierten Geschmack. Trotzdem ist er mit 4 Euro je 100 Gramm deutlich teurer als normaler Früchtetee. ${ }^{18}$

Le mensonge véhiculé par l'image consiste pour l'annonceur à « déléguer » son énonciation à la cible publicitaire que la vue d'un emballage richement illustré va entraîner à une conclusion erronée du type: «On voit des fruits sur l'emballage, donc ce produit contient des fruits ».

15 Le prix a été décerné au Soda « Capri-Sonne » en 2012 et à « Hipp-Kindertee » en 2013.

16 Voir la directive 2000/13/CE du 20 mars 2000 « relative au rapprochement des législations des États membres concernant l'étiquetage et la présentation des denrées alimentaires ainsi que la publicité faite à leur égard ». http://eur-lex.europa.eu/LexUriServ/LexUriServ.do?uri=OJ:L:2000:109:0029:0042: FR:PDF

${ }^{17}$ L'affaire qui mettait en cause la société Spanghero de Castelnaudary avait éclaté en janvier 2013 et s'était soldée par la fermeture de l'entreprise.

${ }^{18} \mathrm{http}: / / w w w . a b g e s p e i s t . d e /$ landlust/index_ger.html 


\begin{tabular}{|c|c|c|}
\hline émetteur & $\begin{array}{c}\text { perception visuelle } / \\
\text { interprétation }\end{array}$ & récepteur \\
\hline image à caractère trompeur & & $\begin{array}{c}\text { énonciation } \\
\text { du contenu trompeur }\end{array}$ \\
\hline
\end{tabular}

Une autre manière de déléguer l'acte de mensonge en faisant porter au destinataire la responsabilité de son énonciation consiste à dissimuler le contenu trompeur dans la partie implicite du message linguistique. Le slogan suivant fonctionne sur ce principe :

(7) Clausthaler, das Bier unter den Alkoholfreien (2009) ${ }^{19}$

La séquence, qui se compose du nom du produit auquel est apposé un groupe nominal complexe, ne fait pas l'objet d'une assertion explicite, mais elle incite le destinataire à reconstruire par inférence plusieurs assertions souhaitées par l'annonceur : 1) Clausthaler est une bière, 2) elle est sans alcool, 3) les autres boissons sans alcool (« die Alkoholfreien ») ne sont pas des bières. L'emploi de l'adjectif substantivé «d- Alkoholfrei- » n'est pas innocent et a d'ailleurs fait l'objet d'une campagne de protestation de la part de l'association Foodwatch ${ }^{20}$ qui considère comme mensonger le fait de qualifier de bière "sans alcool » une boisson qui en contient $0,45 \%$. La législation européenne l'autorise (jusqu'à 1,2\%), mais le terme exact serait selon Foodwatch « alkoholarm » (à faible teneur en alcool).

Un autre aspect discutable, non mentionné par Foodwatch, concerne le dénigrement indirect des marques concurrentes de bières dites «sans alcool», auxquelles ce slogan dénie implicitement le droit de porter le nom de « bière », un contenu habilement induit par le groupe prépositionnel « unter den Alkoholfreien » à valeur sélective incident à la description définie « das Bier ».

Dans l'absolu, l'emploi du terme «bière" pourrait d'ailleurs être remis en cause pour toute boisson à faible teneur en alcool, Clausthaler comprise, quelles que soient ses qualités gustatives. Il faudrait en effet se demander s'il est vraiment légitime (bien qu'aujourd'hui légal) que cette dénomination s'applique à un produit normalement alcoolisé lorsqu'il se trouve privé de ce qui en constitue une caractéristique essentielle (son degré d'alcool).

Dans l'exemple qui vient d'être analysé, tout ce qui peut être contesté et qualifié de trompeur, sinon de mensonger, est exprimé sous forme implicite, mais ce n'est pas toujours le cas comme on va le voir à présent.

\section{Exagération et hyperbole}

Une alternative aux mensonges reposant sur des stratégies de diversion consiste à faire un éloge exagéré du produit, et donc à pousser le curseur au-delà du mensonge.

\footnotetext{
19 http://www.clausthaler-genuss.de/

${ }^{20} \mathrm{http}: / /$ www.abgespeist.de/clausthaler/index_ger.html
} 
Certes, les publicitaires prennent des risques lorsqu'ils affirment explicitement que tel produit possède telle merveilleuse qualité ou engendre tel effet extraordinaire:

(8) Aix en Provence guérit la phlébite (Casino municipal, vers 1910) ${ }^{21}$

Si l'objet vanté ne produit pas l'effet explicitement décrit, le client pourra se sentir trahi et manifester son mécontentement, à moins que la promesse ne soit tellement démesurée qu'on ne puisse plus raisonnablement la prendre au pied de la lettre:

(9) Le pneu Michelin boit l'obstacle (vers 1898) 22

(10) Ala: Le détergent glouton! Ses multi-enzymes dévorent les taches $(1969)^{23}$

Si les situations de la vie quotidienne sont systématiquement idéalisées en publicité (personnages plus beaux, plus jeunes, cadre de vie plus luxueux...), à tel point que les consommateurs n'y prêtent généralement plus attention, l'hyperbole publicitaire pousse la logique plus loin en désamorçant dans la foulée toute critique sur la nature trompeuse de l'exagération: il n'y a plus d'intention trompeuse lorsque le caractère décalé, irréaliste, voire surréaliste de la situation présentée ou de la promesse associée à la campagne devient évident. Catherine Kerbrat-Orecchioni mentionne à ce propos un spot publicitaire des années 80 pour les valises de la marque Samsonite, qui avait été accusée par la société concurrente Delsey de publicité mensongère. On y voyait des bulldozers jouer au football avec une valise Samsonite sans la détruire. Le spot s'achevait sur ce message en voix off:

(11) Samsonite, voyagez tranquille $(1981)^{24}$

L'annonceur avait été condamné en première instance, mais ce jugement avait été invalidé par la Cour d'appel, qui avait jugé qu'une telle hyperbole publicitaire n'était pas de nature à tromper le consommateur (KERBRAT-ORECCHIONI 1986: 337).

L'exagération de la promesse est aussi l'autre face de la dissimulation dont il a été question dans les exemples (2) à (6); les compagnies aériennes, soucieuses de diffuser une image lisse et épurée d'un moyen de transport bruyant et polluant, y ont volontiers recours :

(12) Lufthansa: Fliegen in der Luft, Vögeln gleich (1975) $)^{25}$

(13) Air France: Gagner le cœur du monde (1998) ${ }^{26}$

Le recours à une forme infinitive, qui désactualise le procès, dispense l'annonceur de tout engagement clair quant à la réalisation de la promesse. Peu importe qu'il s'agisse d'un idéal inatteignable. L'actuel slogan d'Air France fonctionne sur le même principe:

(14) Faire du ciel le plus bel endroit de la terre (1999) ${ }^{27}$

\footnotetext{
${ }^{21} \mathrm{http}: / /$ feguide.free.fr/affiche.htm

$22 \mathrm{http} / / /$ commons.wikimedia.org/wiki/File:Michelin_Poster_1898.jpg Voir aussi: LeLIEUR 1999: 293.

23 BArgiel, 2007: 91

${ }^{24} \mathrm{http}: / /$ www.ina.fr/video/PUB3250586025

25 http://www.slogans.de/slogans.php?BSelect[]=283

${ }^{26} \mathrm{http}: / /$ memoireairfrance.canalblog.com/archives/2007/05/07/4865108.html

${ }^{27} \mathrm{http}$ //corporate.airfrance.com/fileadmin/dossiers/documents/communiques_de_presse/Lenvol_ FR.pdf
} 
Ce slogan a été illustré en 2011 par un film publicitaire intitulé «L'envol » où un couple semble danser entre ciel et terre sur un concerto de Mozart ${ }^{28}$. À côté de sa construction infinitive, le slogan comporte une autre spécificité sur le plan grammatical, à savoir l'emploi de la forme superlative qui permet de souligner les qualités exceptionnelles et inégalées du produit vanté (SCHNITZER 2014: 104-105). L'usage de structures comparatives est bien ancré dans la tradition publicitaire:

(15) Pneu Hutchinson: Plus solide que l'acier (affiche de 1920) ${ }^{29}$

Mais en (14), à la différence de (15), la comparaison ne porte pas sur le produit lui-même, mais sur le ciel dont l'extrême beauté n'a pas à être prouvée. Le ciel a-t-il besoin d'Air France pour devenir le plus bel endroit de la terre? Ce que les publicitaires appellent couramment la «promesse » de la marque n'a donc qu'un lointain rapport avec une promesse au sens linguistique. Cet acte de langage est rarement accompli par le biais des slogans qui se contentent le plus souvent d'orienter l'interprétation tout en laissant au récepteur le soin de tirer les conclusions souhaitées.

On a vu que des éléments mensongers, ou tout au moins de nature à induire le consommateur en erreur, étaient couramment véhiculés par l'association des constituants textuels et iconographiques de la publicité, mais il n'est pas rare qu'ils soient directement inscrits dans le nom des produits. Des noms qui apparaissent comme les prémisses des mensonges développés ensuite dans les campagnes de publicité.

\section{Mensonge et dénomination}

La question de la dénomination se pose dès le lancement d'un produit, le nom jouant un rôle non négligeable dans le succès ou l'échec de la mise sur le marché. Dans cette mesure, le choix du nom s'inscrit déjà dans une démarche commerciale. On le constate pour de nombreux modèles de voitures dont les noms sont des promesses d'évasion, d'aventure, de grands espaces :

(16) Jeep Grand Cherokee, Opel Agila, Porsche Cayenne, Renault Captur, Seat Ibiza, Toyota Avensis, etc.

La même logique s'applique à certains parfums aux noms évocateurs, sans rapport avec la composition des parfums mais qui confèrent à ces produits une aura de mystère, de sensualité :

(17) Allure de Chanel, Amazone d'Hermès, Byzance de Rochas, Opium d'Yves Saint-Laurent, etc.

28 Cette chorégraphie très réussie contribue à la mémorisation du slogan par l'association sonimage-texte. http://www.dailymotion.com/video/xl57fk_pub-air-france-envol-2011-hq_creation

29 ftp://ftp.bnf.fr/901/N9011313_PDF_1_-1DM.pdf 
Les vertus réelles ou supposées, pour ne pas dire fantasmagoriques, des crèmes, huiles, parfums et autres produits cosmétiques s'expriment également par le biais de groupes nominaux lexicalisés de forme " adjectif épithète + nom générique » ou assimilée, ou encore de noms composés :

(18) Huile Prodigieuse ou Crème Nirvanesque de Nuxe, Huile Extraordinaire ou Excellence Crème de L'Oréal, Crème Précieuse de l'Occitane, Teint Miracle de Lancôme, Wunderwasser de 4711 Kölnisch Wasser, etc.

En faisant de ces expressions des marques déposées (et protégées), les fabricants s'affranchissent de toute obligation de sincérité: Nuxe n'a pas à démontrer scientifiquement les effets prodigieux de sa crème. Kölnisch Wasser n'est pas non plus tenu d'expliquer ce que sa nouvelle eau de toilette baptisée "Wunderwasser » a de miraculeux. Le nom de marque assimilé à un nom propre possède un caractère conventionnel qui, dans la plupart des cas, le met à l'abri d'éventuelles poursuites.

Dans le secteur alimentaire, la réglementation se révèle néanmoins plus contraignante en matière de dénomination. Depuis les années 90, la marque Danone proposait ainsi à ses clients peu avisés des yaourts «Bio» et tirait avantage de la connotation positive associée à ce terme.

(19) Danone Bio: Yaourt au bifidus actif (1992) ${ }^{30}$

En 2005, pour se mettre en conformité avec la législation européenne ${ }^{31}$, la marque Danone a dû débaptiser ses yaourts « Bio », qui n'avaient de bio que le nom, pour les rebaptiser « $\operatorname{Activia}^{32}$ ».

La guerre des noms ne concerne pas seulement les marques déposées, mais aussi les noms de variétés de produits agricoles autorisées au niveau européen. Les lobbies agroalimentaires, très actifs auprès des instances européennes, parviennent parfois à imposer des dénominations trompeuses, comme en témoigne l'affaire des « fausses échalotes » :

(20) Rose Lily: L'échalote française issue de semis ${ }^{33}$

Les producteurs français d'échalotes traditionnelles sont actuellement en conflit avec des producteurs d'échalotes dites « de semis » venues des Pays-Bas, plus faciles à cultiver, donc vendues moins cher; ces échalotes seraient en réalité une variété d'oignons appelée aussi «échalions » qui n'aurait de l'échalote que l'apparence ${ }^{34}$. Les producteurs d'échalotes traditionnelles considèrent qu'il s'agit d'une contrefaçon et ils ont demandé aux autorités européennes la radiation de l'échalote de semis des catalogues officiels; les consommateurs quant à eux n'y ont vu que du feu. On est

\footnotetext{
${ }^{30}$ http://www.ina.fr/video/PUB3774382002

31 Il s'agissait du règlement $(\mathrm{CEE}) \mathrm{n}^{\circ}$ 2092/91, abrogé entre-temps par le règlement $(\mathrm{CE}) \mathrm{n}^{\circ}$ 834/2007 du 28 juin 2007 « relatif à la production biologique et à l'étiquetage des produits biologiques ».

http://eur-lex.europa.eu/LexUriServ/LexUriServ.do?uri=OJ:L:2007:189:0001:0023:FR:PDF

$32 \mathrm{http} / / /$ lexpansion.lexpress.fr/entreprises/danone-change-ses-bio-en-activia_1360364.html

$33 \mathrm{http} / / /$ www.bejo.fr/fr/actualites/actualit\%C3\%A9s-produits.aspx?PID $=1275 \&$ Action=1\&News $\mathrm{Id}=545$

${ }^{34}$ L'argumentaire des producteurs est présenté dans ce dossier de presse: http://www. echalotetraditionnelle.com/medias/1251901680.pdf
} 
ici en présence d'un cas intéressant de déconnexion entre un signe linguistique et son référent extralinguistique à des fins commerciales. Le procédé consiste à priver un objet du monde du signe linguistique qui lui est habituellement affecté pour le remplacer par un autre dans le but de tromper l'interlocuteur sur la nature même de l'objet. Il s'agit d'une manœuvre subversive sur le plan linguistique puisqu'elle sape le principe même de la dénomination qui s'appuie sur des correspondances stables entre les signes linguistiques et leurs référents. Cette nouvelle dénomination peut avoir pour effet, à moyen terme, de modifier le sens des mots. Si la requête des producteurs d'échalotes traditionnelles n'aboutit pas, le terme « échalote " sera bientôt associé à un végétal aux propriétés botaniques et gustatives différentes de ce qu'était l'échalote avant l'arrivée massive sur le marché de ce nouveau produit.

\section{Appellations et indications d'origine}

On a vu que le contenu trompeur pouvait porter sur différents aspects du produit, de son mode de production à ses effets (souhaités ou indésirables), en passant par ses qualités intrinsèques ou sa composition. Mais la question de l'origine géographique est un point particulièrement sensible, notamment en ce qui concerne les denrées alimentaires, comme l'illustre cette querelle germano-polonaise autour d'une pâtisserie dénommée:

(21) Kołocz śląski/kołacz śląski (Schlesischer Streuselkuchen)

L'enregistrement en 2011 de cette appellation comme indication géographique protégée (IGP) ${ }^{35}$ au bénéfice des boulangers-pâtissiers polonais a eu pour conséquence d'interdire à leurs homologues allemands originaires de Silésie l'utilisation du nom «Schlesischer Streuselkuchen». Ce qui n'était, semble-t-il, qu'une maladresse de traduction a été amplement relayé dans la presse allemande au cours de l'automne $2013^{36}$.

$\mathrm{Si}$ cet exemple reste anecdotique et peu préjudiciable au consommateur, il montre que pour le législateur comme pour tout un chacun, l'enfer est souvent pavé de bonnes intentions. La mise en place d'appellations d'origine protégée ${ }^{37}$ (AOP) et d'indications géographiques protégées ${ }^{38}$ (IGP) a malgré tout permis dans de nombreux cas de limiter la concurrence déloyale et de soutenir la fabrication selon des méthodes traditionnelles de spécialités locales et régionales ${ }^{39}$. Entre 1996

\footnotetext{
${ }^{35}$ Règlement d'exécution (UE) no 733/2011 du 22 juillet 2011 : http://eur-lex.europa.eu/LexUriServ/ LexUriServ.do?uri=OJ:L:2011:195:0032:0033:FR:PDF

${ }^{36}$ On peut citer cet article de Die Welt dont le titre souligne le caractère polémique de l'affaire: "Wie Schlesisch darf deutscher Streuselkuchen sein» http://www.welt.de/121759165, ou encore Der Spiegel, qui prend la chose avec humour en titrant « Versemmelt » http://www.spiegel.de/spiegel/ print/d-113750751.html

37 «Ursprungsbezeichnungen ».

38 « geographische Angaben ».

39 Voir le règlement (UE) $\mathrm{N}^{\circ} 1151 / 2012$, article 5 : « Exigences applicables aux appellations d'origine et aux indications géographiques ».

http://eur-lex.europa.eu/LexUriServ/LexUriServ.do?uri=OJ:L:2012:343:0001:0029:fr:PDF
} 
et 2013, pas moins de 206 AOP ont été enregistrées côté français, 95 côté allemand ${ }^{40}$, de la « gâche vendéenne » à la « figue de Solliès », du « Spargel aus Franken » au « Lübecker Marzipan ».

Il n'en reste pas moins extrêmement difficile de statuer sur le caractère trompeur, voire mensonger de la dénomination de telle spécialité non enregistrée au catalogue. On n'a pas le droit d'appeler «roquefort» un fromage fabriqué en Bretagne, mais rien n'empêche d'apposer l'étiquette «camembert» sur un fromage venu de Hollande, du moment qu'on ne précise pas « camembert de Normandie », puisque le nom « camembert » n'est pas protégé. Sur le plan légal, cette forme d'usurpation d'identité, qu'on pourrait qualifier d'usurpation de signifiant sur le plan linguistique, n'est pas considérée comme trompeuse et ne donne lieu à aucune sanction.

\section{Conclusion}

Les stratégies couramment utilisées par les marques pour tromper le consommateur, tout en restant dans les limites d'un cadre légal toujours plus contraignant, vont du mensonge par omission à l'hyperbole en passant par la désactualisation de la promesse, quand elles ne visent pas la dénomination elle-même. Les moyens de contourner les directives européennes sur la publicité trompeuse sont multiples et perpétuellement renouvelés au gré de l'évolution de la législation. Le mensonge est parfois directement exprimé, par le biais d'une construction assertive, parfois à reconstruire par le destinataire, lorsqu'il est véhiculé par l'image ou implicitement contenu dans l'énoncé. Indétectable en langue, idéalement transparent dans le discours, la présence du mensonge se manifeste à travers certains indices que l'analyse du corpus a contribué à mettre en évidence. Tous les supports linguistiques sont capables d'accueillir des mensonges effectifs ou par délégation, mais on a vu que dans le domaine de la communication commerciale, les tournures infinitives, les superlatifs, les phrases sans verbes, ou encore les dénominations constituaient un terreau particulièrement favorable.

\section{Bibliographie}

Bargiel Réjane, 150 ans de publicité, Paris, Les arts décoratifs, 2007.

HundSNURSCHER Franz, «Lügen - auch eine Form sprachlichen Handelns », in Dieter Halwachs, Christine Penzinger, Irmgard Stütz (dir.), Sprache, Onomatopöie, Rhetorik, Namen, Idiomatik, Grammatik, Graz, Karl-Franzens-Universität Graz (coll. « Grazer Linguistische Monographien », 11), 1994, p. 97-113.

www.uni-graz.at/ling2www_glm11_hundsnurscher.pdf

JANICH Nina, Werbesprache. Ein Arbeitsbuch, Tübingen, Narr, 1999.

LEFEBVRE Thierry, «L'Urodonal et ses publicités », Revue d'histoire de la pharmacie, n 344 , 2004, p. 680-684.

${ }^{40}$ La base de données DOOR est consultable sur le site de la commission Européenne.

http://ec.europa.eu/agriculture/quality/door/list.html 
http://www.persee.fr/web/revues/home/prescript/article/pharm_0035-2349_2004_ num_92_344_5751

LENCLUD Gérard, «L'acte de mentir », Terrain, $\mathrm{n}^{\circ}$ 57, 2011, p. 4-19.

Kerbrat-Orecchioni Catherine, L'implicite, Paris, Armand Colin, 1986.

KüMmerling-Meibauer Bettina, Meibauer, Jörg, «Lügenerwerb und Geschichten vom Lügen », Zeitschrift für Literaturwissenschaft und Linguistik (LiLi), n 62, 2011, p. 114-134.

http://homepages.uni-tuebingen.de/bettina.kuemmerling-meibauer/essays/Luegenerwerb_ und_Geschichten_vom_Luegen.pdf

Lelieur Anne-Claude (dir.), De Bébé Cadum à Mamie Nova. Un siècle de personnages publicitaires, Paris, Paris-bibliothèques, 1999.

Reboul Anne, «Le paradoxe du mensonge dans la théorie des actes de langage », Cahiers de linguistique française, $\mathrm{n}^{\circ}$ 13, 1992, p. 125-147.

SCHNitzer Nathalie, "Wie ein Star" - Vergleich und Präsupposition in der Werbung », in Michel LefÈVRe (dir.), Linguistische Aspekte des Vergleichs, der Metapher und der Metonymie, Tübingen, Stauffenburg-Verlag, 2014, p. 103-117. 